\title{
ON CENTRAL TRACES AND GROUPS OF SYMMETRIES OF ORDER UNIT BANACH SPACES
}

\author{
by CHO-HO CHU(*) \\ (Received 3rd March 1977)
}

\section{Introduction}

A central trace on an order-unit Banach space $A(K)$ is a centre-valued module homomorphism invariant under the group of symmetries of $A(K)$.

The concept of central traces has been crucial in the theory of types for convex sets established in (4), (5). In von Neumann algebras, they are precisely the canonical centre-valued traces and their existence hinges on a fundamental theorem (Dixmier's approximation process) in von Neumann algebras. On the other hand, the existence of central traces in finite dimensional spaces is an easy consequence of Ryll-Nardzewski's fixed point theorem (5).

The main objective of this note is to demonstrate, by means of the Bochner integral, that if a group of symmetries is uniformly almost periodic, then there is a positive operator on $A(K)$ which resembles a central trace. In particular, if the group of all symmetries is uniformly almost periodic as well as transitive and also, if $K$ satisfies Størmer's axiom, then there is a central trace on $A(K)$. The results proved here for compact convex sets, extending some of those in (8) on topological dynamics and $C^{*}$-algebras, appear to be more unified and transparent.

\section{Central traces and groups of symmetries}

Let $K$ be a compact convex subset of a locally convex space and let $A(K)$ be the Banach space of continuous affine functions on $K$ with the constant 1 -function $e$ as order-unit. We shall refer to (1) for undefined terminology.

$K$ is said to satisfy Størmer's axiom if the closed convex hull $\overline{c o} \cup_{\alpha} F_{\alpha}$ of a family $\left\{F_{\alpha}\right\}$ of closed split faces of $K$ is also a split face. The set of extreme points of $K$ will be denoted by $\partial K$. For each extreme point $k$, let $F_{k}$ be the smallest closed split face of $K$ containing $k$. As usual, $K$ will be identified, whenever it is convenient, with the vaguely compact convex state space $\left\{\varphi \in A(K)^{*}: \varphi(e)=\|\varphi\|=1\right\}$ of $A(K)$.

Let $O(A)$ denote the set of all order-bounded linear operators on $A(K)$, that is all linear operators $T: A(K) \rightarrow A(K)$ such that $-\|T\| I \leqslant T \leqslant\|T\| I$ where $I$ is the identity operator on $A(K)$. The set $Z=\{T e: T \in O(A)\}$ is the centre of $A(K)$. For each $a$ in $A(K)$ and $T$ in $O(A)$, we have

$$
(T a)(k)=(T e)(k) \cdot a(k)
$$

where $k$ is any extreme point of $K$.

(*) Work done at the University of Tripoli. 
A symmetry of $A(K)$ is an isometric order-isomorphism $\sigma: A(K) \rightarrow A(K)$ such that $\sigma(e)=e$ and $\sigma T=T \sigma$ for each $T$ in $O(A)$. A central trace on $A(K)$ is a positive linear operator $\Gamma: A(K) \rightarrow Z$ such that $\Gamma(e)=e, T \Gamma=\Gamma T$ for each $T$ in $O(A)$ and, $\Gamma \sigma=\Gamma$ for each symmetry $\sigma$ of $A(K)$ (See (4) and (5)).

The set $S$ of all symmetries of $A(K)$ form a group with respect to function composition. The identity of $S$ is the identity operator $I$ on $A(K)$. For each $\sigma$ in $S$, we let $\sigma^{*}: A(K)^{*} \rightarrow A(K)^{*}$ be the dual of $\sigma$; then $\sigma^{*}$ restricts to an affine homeomorphism from $K$ onto itself. We shall also denote by $\sigma^{*}$ this restriction to $K$ if no confusion will arise.

Let $G$ be a group of symmetries of $A(K)$ and let $A^{A}$ denote the product space $A(K)^{A(K)}$ with the product topology so that a net $\left\{\sigma_{\alpha}\right\}$ in $G \subseteq A^{A}$ converges to an element $\tau$ in $A^{A}$ if and only if $\left\|\sigma_{\alpha}(a)-\tau(a)\right\| \rightarrow 0$ for each $a$ in $A(K)$. The uniform boundedness principle shows that $\tau: A(K) \rightarrow A(K)$ is a positive bounded operator with $\|\tau\| \leqslant 1$ and also $\tau^{*} K \subseteq K$. Moreover, $G$ is a topological group in the product topology. In fact, it is easily seen that the group multiplication is continuous. Further, if $\sigma_{\alpha} \rightarrow \sigma$ in $G$, then by isometry, for each $a$ in $A(K),\left\|\sigma_{\alpha}^{-1} a-\sigma^{-1} a\right\|=\left\|\sigma_{\alpha}^{-1}\left(a-\sigma_{\alpha} \sigma^{-1} a\right)\right\|=$ $\left\|a-\sigma_{\alpha} \sigma^{-1} a\right\|=\left\|\sigma\left(\sigma^{-1} a\right)-\sigma_{\alpha}\left(\sigma^{-1} a\right)\right\| \rightarrow 0$. So the inverse operation is also continuous on G.

Next the action $K \times G \rightarrow K:(k, \sigma) \rightarrow \sigma^{*}(k)$ of $G$ on $K$ makes $(K, G)$ into a transformation group. We say that $G$ acts transitively on $K$ if for each extreme point $k, \partial F_{k}$ is contained in the closure of $\left\{\sigma^{*}(k): \sigma \in G\right\}$, or equivalently, if $F_{k}=$ $\overline{\operatorname{co}}\left\{\sigma^{*}(k): \sigma \in G\right\}$.

Plainly, if every extreme point of $K$ is a split face, then any group $G$ of symmetries is transitive since for each $k$ in $\partial K, F_{k}=\{k\}$ and for each $\sigma$ in $G, k=\sigma^{*} x$ for some $x$ in $\partial K$.

Example 1. Let $K$ be the state space of a $C^{*}$-algebra $A$ with identity. Then $A(K)$ can be identified with the self-adjoint part of $A$. It is evident that the inner automorphisms of $A$ are symmetries. Indeed using a theorem of Kadison (9, Theorem 10), one can show that the symmetries of $A(K)$ are just (the real parts of) the automorphisms of $A$ leaving the centre of $A$ fixed (see (5)). Moreover, it also follows from Kadison's transitivity theorem (10) that $S$ acts transitively on $K$.

Let $G$ be a group of symmetries of $A(K)$. An element $a$ in $A(K)$ is called $G$-invariant if $\sigma(a)=a$ for all $\sigma$ in $G$. We shall denote by $A_{G}$ the set of $G$-invariant elements in $A(K)$. A subset $F$ of $K$ is called $G$-invariant if $\sigma^{*} F \subseteq F$ for all $\sigma$ in $G$. The set of all $G$-invariant elements in $K$ will be denoted by $K_{G}$ which is clearly a compact convex set.

Let $G_{0}=\left\{\sigma \in S: \sigma^{* *}\right.$ is a symmetry of $\left.A(K)^{* *}\right\}$. The following proposition generalises a well-known result in $C^{*}$-algebras concerning the invariant and split faces.

Proposition 1. Let $G$ be a subgroup of $G_{0}$. Let $F$ be a split face of $K$. Then $F$ is $G$-invariant. Conversely, if $G$ is transitive and $K$ satisfies Størmer's axiom, then every closed $G$-invariant face is a split face.

Proof. Let $F$ be a split face of $K$. Then there is a central projection $E: A(K)^{*} \rightarrow$ 
$A(K)^{*}$ such that $F=E A(K)^{*} \cap K$. Let $\sigma \in G$. Then $\sigma^{* *} E^{*}=E^{*} \sigma^{* *}$ since $\sigma^{* *}$ is a symmetry of $A(K)^{* *}$. It follows that $\sigma^{*} E=E \sigma^{*}$ and hence $\sigma^{*} F \subseteq F$. So $F$ is $G$-invariant.

Conversely, suppose $F$ is a closed $G$-invariant face. Let $k \in \partial F$. Then, by transitivity of $G, F_{k}=\overline{c o}\left\{\sigma^{*}(k): \sigma \in G\right\}$ which is contained in $F$ as $F$ is $G$-invariant. Therefore $F=\overline{c o} \cup_{k \in \partial F} F_{k}$ is a split face by Størmer's axiom.

Example 2. Evidently, $G_{0}=S$ if $K$ is finite dimensional. Let us take $K$ to be a square or a disc in the plane. Then it is not difficult to see that the centre of $K$ is the only element of $K_{S}$.

Example 3. Let $K$ be the state space of a function algebra. Since each extreme point of $K$ is a split face, it follows from the above proposition and the Krein-Milman Theorem that $K=K_{G_{0}}$. Thus $K_{G_{0}}$ need not be a Choquet simplex in general.

Example 4. Let $K$ be the state space of a $C^{*}$-algebra with identity and let $U$ be the group of all inner automorphisms of $A$. Then $(A, U)$ form a $U$-abelian system (see $(14,3.1 .11)$ for definition) and as $U \subseteq G_{0},\left(A, G_{0}\right)$ form a $G_{0}$-abelian system as well. It follows that $K_{G_{0}}$ is a Choquet simplex (see (11)). Note that if $A$ is a type $I$ von Neumann algebra, then $U=S$ (see $(14 ; 2.9 .32)$ ).

It would be interesting to characterise those compact convex sets $K$ for which $K_{G_{0}}$ are Choquet simplexes.

Let us observe that the centre of a $C^{*}$-algebra is the set of elements which are invariant under the inner automorphisms. This is a particular instance of the following more general result in convexity.

Proposition 2. Let $G$ be a transitive group of symmetries of $A(K)$ and let $Z$ be the centre of $A(K)$. Suppose $K$ satisfies Størmer's axiom. Then $Z=A_{G}$.

Proof. Since, for each $z$ in $Z$, there is an order-bounded operator $T$ in $O(A)$ such that $z=T e$, we have $\sigma z=\sigma T e=T \sigma e=T e=z$ for all $\sigma$ in $G$. So $Z$ is contained in $A_{G}$.

Let us recall that $Z$ is identifiable with the space of facially continuous functions on $\partial K$ (see (1)). Now suppose $a \in A(K)$ is $G$-invariant. We show that $a$ is facially continuous on $\partial K$. Let $r, t$ be any real numbers and let $W=\{w \in \partial K: r \leqslant a(w) \leqslant t\}$. We prove that $W$ is facially closed in $\partial K$. Let $F=\overline{c o} \cup_{w \in W} F_{w}$. Then $F$ is a split face by Størmer's axiom. We assert that $W=\partial F$. Let us first prove that $a$ is constant on $\partial F_{w}$ for each $w$ in $W$. In fact, if $k$ is an element in $\partial F_{w}$, then the transitivity of $G$ gives $k \in\left\{\sigma^{*} w: \sigma \in G\right\}^{-}$. But $a$ is $G$-invariant, so $a \sigma^{*}(w)=\sigma a(w)=a(w)$ for all $\sigma$ in $G$. Therefore $a(k)=a(w)$ by continuity. Hence $a$ is a constant on $\partial F_{w}$ and it follows that $\partial F_{w} \subseteq W$. Now the affineness and continuity of a give $\partial F \subseteq W$. Thus $W=\partial F$ as asserted. This proves that $a$ is facially continuous on $\partial K$, that is, $a \in Z$. As $a \in A_{G}$ was arbitrary, we conclude that $Z=A_{G}$.

\section{Uniform almost periodicity}

In this section, we shall apply some ideas from (8) to study the groups of 
symmetries of $A(K)$ and to prove the existence of an analogue of central traces by using the Bochner integral.

Let $G$ be a group of symmetries of $A(K)$. The transformation group $(K, G)$ is said to be uniformly almost periodic if for each $f$ in $C(K), f$ is almost periodic (with respect to $G$ ), that is $\left\{f \sigma^{*}: \sigma \in G\right\}$ has compact closure in $C(K)$ (see (7)).

We first extend a result of Green in (8).

Proposition 3. The transformation group $(K, G)$ is uniformly almost periodic if and only if $G$ has compact closure in $A(K)^{A(K)}$ in the product topology. Under this condition, the closure $G^{-}$is a topological group of symmetries of $A(K)$.

Proof. First suppose $(K, G)$ is uniformly almost periodic. For each $a$ in $A(K)$, let $[a]=\{\sigma a: \sigma \in G\}$ be the orbit of $a$. Then, by uniform almost periodicity, $[a]^{-}=$ $\left\{a \sigma^{*}: \sigma \in G\right\}^{-}$is compact in $C(K)$ and hence compact in $A(K)$. So $\Pi_{a \in A(K)}[a]^{-}$is compact in $A^{A}$ in the product topology. Let $\tau \in G^{-} \subseteq A^{A}$. Then there is a net $\left\{\sigma_{\alpha}\right\}$ in $G$ such that $\left\|\sigma_{\alpha} a-\tau a\right\| \rightarrow 0$ for each $a$ in $A(K)$. Therefore $\tau a \in[a]^{-}$for each $a$ in $A(K)$. It follows that $G^{-} \subseteq \Pi_{a \in A(K)}[a]^{-}$is compact.

Conversely, let $G^{-}$be compact in $A^{A}$. Let us first prove that $\left\{\sigma^{*}: \sigma \in G^{-}\right\}$is compact in $K^{K}$ in the uniform topology, that is, for any net $\left\{\sigma_{\alpha}^{*}\right\}$, there is a subnet $\left\{\sigma_{\beta}^{*}\right\}$ converging to $\tau^{*}$, where $\tau \in G^{-}$, in that

$$
\sup _{k \in K}\left|\sigma_{\beta}^{*}(k)(a)-\tau^{*}(k)(a)\right| \rightarrow 0
$$

for each $a$ in $A(K)$. As $G^{-}$is compact in $A^{A}$, there is a subnet $\left\{\sigma_{\beta}\right\}$ of $\left\{\sigma_{\alpha}\right\}$ converging to an element $\tau \in G^{-}$in the product topology. It follows that $\sup \mid \sigma_{\beta}^{*}(k)(a)-$ $\tau^{*}(k)(a)|=\sup | \sigma_{\beta}(a)(k)-\tau(a)(k) \mid \leqslant\left\|\sigma_{\beta}(a)-\tau(a)\right\| \rightarrow 0$. This proves that $\left\{\sigma^{*}: \sigma \in G^{-}\right\}$ is compact in $K^{K}$ in the uniform topology and so it is equicontinuous (2; Corollary 3 p. 292). Hence $\left\{\sigma^{*}: \sigma \in G\right\}$ is also equicontinuous in $K^{K}$. It follows that $\left\{f \sigma^{*}: \sigma \in G\right\}$ has compact closure in $C(K)$ for each $f$ in $C(K)(7 ; 4.4,4.5)$. Therefore $(K, G)$ is uniformly almost periodic.

Finally we show that $G^{-}$is contained in the group $S$ of all symmetries of $A(K)$ under this condition. Let $\tau \in G^{-}$and let $\left\{\sigma_{\alpha}\right\}$ be a net in $G$ converging to $\tau$. We have noted before that $\tau$ is a positive bounded operator on $A(K)$. Let us show that $\tau$ has an inverse. Indeed, by compactness of $G^{-}$, there is a subnet $\left\{\sigma_{\beta}^{-1}\right\}$ of the net $\left\{\sigma_{\alpha}^{-1}\right\}$ converging to some $\sigma$ in $G^{-}$. Therefore $\tau \sigma=\tau \lim _{\beta} \sigma_{\beta}^{-1}=\lim _{\beta} \sigma_{\beta} \sigma_{\beta}^{-1}=I=\sigma \tau$. So $\tau$ has an inverse in $G$. Now it is routine to verify that $\tau$ is a symmetry of $A(K)$. The proof is complete.

Corollary 4. Let $(K, G)$ be uniformly almost periodic. Then $K$ contains a $G$ invariant element. In particular, if $(K, S)$ is uniformly almost periodic and $A(K)$ has trivial centre, then there is a central trace on $A(K)$.

Proof. Since $\left\{\sigma^{*}: \sigma \in G\right\} \subseteq K^{K}$ is an equicontinuous group of affine mappings, Kakutani's fixed point theorem asserts that there is an element $k$ in $K$ such that $\sigma^{*}(k)=k$ for all $\sigma$ in $G$ (see $(6$, p. 457)). Further, if the centre $Z$ of $A(K)$ is trivial, the operator $\Gamma: A(K) \rightarrow Z$ defined by $\Gamma(a)=a(k) e$ is obviously a central trace. 
We now construct a Bochner integral on a uniformly almost periodic group of symmetries.

Theorem 5. Let $G$ be a group of symmetries of $A(K)$ and let $(K, G)$ be uniformly almost periodic. Then there is a positive linear operator $\Gamma: A(K) \rightarrow A_{G}$ satisfying the following conditions:

(i) $\Gamma(e)=e$;

(ii) $\Gamma T=T \Gamma$ for each $T$ in $O(A)$;

(iii) $\Gamma \sigma=\Gamma$ for each $\sigma$ in $G$.

Proof. By Proposition $3, G^{-}$is a compact topological group. Let $\mu$ be the normalised Haar measure on $G^{-}$. For each $a$ in $A(K)$, the continuous function $\sigma \mapsto \sigma(a): G^{-} \rightarrow A(K)$ is separably-valued since $G^{-}$is compact. Also, it is clearly weakly measurable. Furthermore, the real function $\sigma \mapsto\|\sigma(a)\|$ is bounded and hence $\mu$-integrable on $G^{-}$. It follows that $\sigma \mapsto \sigma(a)$ is Bochner $\mu$-integrable (16; Theorem 1, p. 133). Let

$$
\Gamma(a)=\int_{G^{-}} \sigma(a) d \mu(\sigma)(a \in A(K))
$$

be the Bochner integral. Then, by (16; Corollary 2, p. 134), for each $\tau$ in $G$, we have $\tau(\Gamma a)=\tau \int_{G^{-}} \sigma(a) d \mu(\sigma)=\int_{G^{-}} \tau \sigma(a) d \mu(\sigma)=\int_{G^{-}} \sigma(a) d \mu(\sigma)=\Gamma(a)$ by the invariance of $\mu$. This shows that $\Gamma(a)$ is $G$-invariant, that is, $\Gamma(a) \in A_{G}$.

It is evident that $\Gamma: A(K) \rightarrow A_{G}$ is positive and linear. To complete the proof, it remains to verify conditions (i), (ii) and (iii). First, condition (i) follows easily from the fact that $\mu$ is normalised. Next, let $T \in O(A)$, then again by (16; Corollary 2, p. 134), we have $T \Gamma(a)=T \int_{G^{-}} \sigma(a) d \mu(\sigma)=\int_{G^{-}} T \sigma(a) d \mu(\sigma)=\int_{G^{-}} \sigma(T a) d \mu(\sigma)=\Gamma(T a)$. So $T \Gamma=\Gamma T$. Finally, as before, the invariance of $\mu$ implies that $\Gamma \sigma=\Gamma$ for each $\sigma$ in $G$. This concludes the proof.

Corollary 6. Let $S$ be the group of all symmetries of $A(K)$. Suppose that $(K, S)$ is transitive, uniformly almost periodic and also, $K$ satisfies Stormer's axiom. Then there is a central trace on $A(K)$.

Proof. By Proposition 2, $A_{S}$ is the centre of $A(K)$ and so the operator $\Gamma$ in Theorem 5 is a central trace on $A(K)$.

We shall give some examples at the end of the paper.

\section{Tensor products}

We consider in this section the tensor products of uniformly almost periodic groups. We shall use (13) and (15) as our references to tensor products of compact convex sets. Let $K_{1}$ and $K_{2}$ be two compact convex sets and let $K_{1} \otimes K_{2}$ be their injective tensor product. Let us recall that each extreme point of $K_{1} \otimes K_{2}$ is the product $k_{1} \otimes k_{2}$ of two uniquely determined extreme points $k_{1}$ of $K_{1}$ and $k_{2}$ of $K_{2}$ (cf. (13)). The injective tensor product of $A\left(K_{1}\right)$ and $A\left(K_{2}\right)$ will be denoted by $A\left(K_{1}\right) \otimes A\left(K_{2}\right)$ which is identified as the space $A\left(K_{1} \otimes K_{2}\right)$ of continuous affine 
functions on $K_{1} \otimes K_{2}$ with $e_{1} \otimes e_{2}$ as the constant 1-function where $e_{1}$ is the order-unit in $A\left(K_{1}\right)$ and $e_{2}$ the order-unit in $A\left(K_{2}\right)$.

Next if $\sigma_{1} ; A\left(K_{1}\right) \rightarrow A\left(K_{1}\right)$ and $\sigma_{2}: A\left(K_{2}\right) \rightarrow A\left(K_{2}\right)$ are bounded operators, we define $\sigma_{1} \otimes \sigma_{2}: A\left(K_{1}\right) \otimes A\left(K_{2}\right) \rightarrow A\left(K_{1}\right) \otimes A\left(K_{2}\right)$ to be the unique bounded operator on $A\left(K_{1}\right) \otimes A\left(K_{2}\right)$ such that $\left(\sigma_{1} \otimes \sigma_{2}\right)\left(a_{1} \otimes a_{2}\right)=\sigma_{1}\left(a_{1}\right) \otimes \sigma_{2}\left(a_{2}\right)$ for $a_{1} \in A\left(K_{1}\right)$ and $a_{2} \in$ $A\left(K_{2}\right)$ (cf. 15).

Proposition 7. Let $\sigma_{1}: A\left(K_{1}\right) \rightarrow A\left(K_{1}\right)$ and $\sigma_{2}: A\left(K_{2}\right) \rightarrow A\left(K_{2}\right)$ be symmetries. Then $\sigma_{1} \otimes \sigma_{2}: A\left(K_{1}\right) \otimes A\left(K_{2}\right) \rightarrow A\left(K_{1}\right) \otimes A\left(K_{2}\right)$ is a symmetry.

Proof. By the functorial property of the injective tensor products $(15 ;$ p. 74), $\sigma_{1} \otimes \sigma_{2}$ is an order-isometric isomorphism and also, $\left(\sigma_{1} \otimes \sigma_{2}\right)\left(e_{1} \otimes e_{2}\right)=\sigma_{1} e_{1} \otimes \sigma_{2} e_{2}=$ $e_{1} \otimes e_{2}$. It remains to prove that $\sigma_{1} \otimes \sigma_{2}$ commutes with the order-bounded operators. Let $T: A\left(K_{1}\right) \otimes A\left(K_{2}\right) \rightarrow A\left(K_{1}\right) \otimes A\left(K_{2}\right)$ be an order-bounded operator. Then by (3, Proposition 9), we may assume that there exist order-bounded operators $P_{n}: A\left(K_{1}\right) \rightarrow$ $A\left(K_{1}\right)$ and $Q_{n}: A\left(K_{2}\right) \rightarrow A\left(K_{2}\right)$ such that

$$
\left\|P_{n} e_{1} \otimes Q_{n} e_{2}-T\left(e_{1} \otimes e_{2}\right)\right\| \rightarrow 0 \text { as } n \rightarrow \infty .
$$

Let $f$ be an element of $A\left(K_{1}\right) \otimes A\left(K_{2}\right)$. Then we have

$$
\begin{aligned}
\|\left(P_{n} \otimes Q_{n}\right)(f) & -T f \|=\sup _{k_{1} \otimes k_{2} \in a\left(K_{1} \otimes K_{2}\right)}\left|\left(P_{n} \otimes Q_{n}\right)(f)\left(k_{1} \otimes k_{2}\right)-(T f)\left(k_{1} \otimes k_{2}\right)\right| \\
& =\sup \left|\left(P_{n} \otimes Q_{n}\right)\left(e_{1} \otimes e_{2}\right)\left(k_{1} \otimes k_{2}\right) f\left(k_{1} \otimes k_{2}\right)-T\left(e_{1} \otimes e_{2}\right)\left(k_{1} \otimes k_{2}\right) f\left(k_{1} \otimes k_{2}\right)\right|
\end{aligned}
$$

by $(2.1)$

$$
\begin{aligned}
& =\sup \left|\left(P_{n} e_{1} \otimes Q_{n} e_{2}\right)\left(k_{1} \otimes k_{2}\right)-T\left(e_{1} \otimes e_{2}\right)\left(k_{1} \otimes k_{2}\right) \| f\left(k_{1} \otimes k_{2}\right)\right| \\
& \leqslant\|f\|\left\|P_{n} e_{1} \otimes Q_{n} e_{2}-T\left(e_{1} \otimes e_{2}\right)\right\| \rightarrow 0 \text { as } n \rightarrow \infty .
\end{aligned}
$$

It follows that

$$
\begin{aligned}
\left(\sigma_{1} \otimes \sigma_{2}\right)(T f) & =\lim _{n}\left(\sigma_{1} \otimes \sigma_{2}\right)\left(P_{n} \otimes Q_{n}\right)(f) \\
& =\lim _{n}\left(\sigma_{1} P_{n} \otimes \sigma_{2} Q_{n}\right)(f) \\
& =\lim _{n}\left(P_{n} \sigma_{1} \otimes Q_{n} \sigma_{2}\right)(f) \\
& =\lim _{n}\left(P_{n} \otimes Q_{n}\right)\left(\sigma_{1} \otimes \sigma_{2}\right)(f) \\
& =T\left(\sigma_{1} \otimes \sigma_{2}\right)(f) .
\end{aligned}
$$

This proves that $\left(\sigma_{1} \otimes \sigma_{2}\right) T=T\left(\sigma_{1} \otimes \sigma_{2}\right)$. Therefore $\sigma_{1} \otimes \sigma_{2}$ is a symmetry.

Let $\left(K_{1}, G_{1}\right)$ and $\left(K_{2}, G_{2}\right)$ be uniformly almost periodic groups. Then $G_{1}^{-}$and $G_{2}^{-}$ are compact topological groups by Proposition 3. Let

$$
G=G_{1} \otimes G_{2}=\left\{\sigma_{1} \otimes \sigma_{2}: \sigma_{1} \in G_{1}, \sigma_{2} \in G_{2}\right\} .
$$

Then it follows from Proposition 7 that $G$ is a group of symmetries of $A\left(K_{1}\right) \otimes A\left(K_{2}\right)$. Let $A=A\left(K_{1}\right) \otimes A\left(K_{2}\right)$. Define a map $G_{1}^{-} \times G_{2}^{-} \rightarrow A^{A}$ by $\left(\sigma_{1}, \sigma_{2}\right) \mapsto \sigma_{1} \otimes \sigma_{2}$. Then a 
simple application of the uniform boundedness principle (6, p. 55) shows that the map is continuous and its range is just $\left(G_{1} \otimes G_{2}\right)^{-}$by compactness of $G_{1}^{-}$and $G_{2}^{-}$. It follows that $\left(G_{1} \otimes G_{2}\right)^{-}$is compact in the product topology. Thus we have proved the following result.

Theorem 8. Let $\left(K_{1}, G_{1}\right)$ and $\left(K_{2}, G_{2}\right)$ be uniformly almost periodic groups. Then $\left(K_{1} \otimes K_{2}, G_{1} \otimes G_{2}\right)$ is a uniformly almost periodic group.

We conclude the paper with a few illustrative examples.

Example 5. Let $K_{1}$ be a Bauer simplex. Then the group $S_{1}$ of symmetries of $A\left(K_{1}\right)$ consists of only one element, namely, the identity operator on $A\left(K_{1}\right)$. So $\left(K_{1}, S_{1}\right)$ is uniformly almost periodic.

Example 6. Let $K_{2}$ be a square in the plane. Then, since there is no non-trivial order-bounded operators on $A\left(K_{2}\right)$, the symmetries $S_{2}$ of $A\left(K_{2}\right)$ are precisely the order-isometric automorphisms. Therefore $\left\{\sigma^{*}: \sigma \in S_{2}\right\}$ consists of all the affine autohomeomorphisms of $K_{2}$ and is just the dihedral group of order 8 (see (12, p. 55)). It follows that $\left(K_{2}, S_{2}\right)$ is uniformly almost periodic.

Example 7. Let $A$ be a UHF algebra and $K_{3}$ its state space. Let $\left\{A_{n}\right\}$ be its defining sequence with $A_{n}=M_{1} \otimes \cdots \otimes M_{n}$ where each $M_{i}$ is a finite dimensional factor. Let $U$ be the group of all unitaries in $\cup_{n=1}^{\infty} A_{n}$ which have the form $u_{1} \otimes \cdots \otimes u_{k}$ where $u_{i}$ is a unitary in $M_{i}$. Let $S_{3}$ be the group of (the real parts of) all inner automorphisms of $A$ induced by the elements of $U$. Then it was shown in (8) that $\left(K_{3}, S_{3}\right)$ is uniformly almost periodic.

Now we can use Theorem 8 to construct a few more uniformly almost periodic groups.

Example 8. In the above examples, the groups $\left(K_{i} \otimes K_{j}, S_{i} \otimes S_{j}\right) i, j=1,2,3$, are uniformly almost periodic.

\section{REFERENCES}

(1) E. M. ALfSEN, Compact convex sets and boundary integrals (Ergebnisse der Math. 57, Springer-Verlag, Berlin, 1971).

(2) N. Bourbaki, General topology, Part 2 (Addison-Wesley, Reading, 1966).

(3) ChO-Ho CHU, On standard elements and tensor products of compact convex sets, $J$. London Math. Soc. 14 (1976), 71-78.

(4) ChO-Ho CHU and J. D. M. WRIGHT, Une théorie des types pour une classe d'éspaces de Banach ordonnés, C.R. Acad. Sc. Paris 281A (1975), 633-636.

(5) CHO-Ho CHU and J. D. M. WRIGHT, A theory of types for convex sets and ordered Banach spaces, Proc. London Math. Soc. 36 (1978), $494-517$. 1958).

(6) N. DUNFORD and J. T. SCHWARTZ, Linear operators, Part I (Interscience, New York, 
(7) R. Ellis, Lectures on topological dynamics (Benjamin, New York, 1969).

(8) W. L. GreEN, Topological dynamics and $C^{*}$-algebras, Trans. Amer. Math. Soc. 210 (1975), 107-121.

(9) R. V. KADISON, Isometries of operator algebras, Ann. of Math. (1951), 325-338.

(10) R. V. Kadison, Irreducible operator algebras, Proc. Nat. Acad. Sc. USA 43 (1957), 273-276.

(11) O. LANFORD and D. RUELLE, Integral representations of invariant states on $B^{*}$. algebras, J. Math. Physics, 8 (1967), 1460-1463.

(12) W. LEDERMANN, Introduction to the theory of finite groups (Oliver and Boyd, Edinburgh, 1967).

(13) I. NAmiokA and R. R. Phelps, Tensor products of compact convex sets, Pacific J. Math. 31 (1969), 469-480.

(14) S. SAKAI, $C^{*}$-algebras and $W^{*}$-algebras (Ergebnisse der Math. 60, Springer-Verlag, Berlin, 1971).

(15) G. WITTSTOCK, Ordered normed tensor products, Foundations of Quantum mechanics and ordered linear spaces ((Marburg) Lecture Notes in Physics 29, Springer-Verlag, Berlin, 1974).

(16) K. YosidA, Functional Analysis (Grundlehren der Math. Wissenchaften, Band 123, Springer-Verlag, Berlin, 1971).

UNIVERSITY OF BENIN

BENIN CITY

NIGERIA 